\title{
Correlating analytical microscopy reveals quantitative alterations to the structure, chemistry and materials properties of tooth enamel exposed to acidic solutions.
}

\author{
Louise Hughes ${ }^{1}$, Iain Anderson ${ }^{1}$ and Jonathan Moffat ${ }^{2}$ \\ ${ }^{1}$ Oxford Instruments NanoAnalysis, United States, ${ }^{2}$ Oxford Instruments Asylum Research, United States
}

Analysing quantitative data from correlative microscopy techniques is of increasing interest to the research community, with many more imaging modalities being incorporated into correlative workflows [1]. Recording dynamic changes using more than one microscopy technique is also a significant challenge, involving reimaging the same region more than once with any chosen modality. We have used correlative microscopy to record changes in tooth enamel structure, chemistry, mechanical properties, and topography following exposure to acidic solutions using electron microscopy, energy dispersive x-ray spectrometry (EDS) and atomic force microscopy (AFM), respectively.

Deer teeth were prepared using a diamond saw and wet sanding to generate a thick cross section through the centre of the teeth. The final polishing was done manually using 3000 grit wet sandpaper. The teeth were irrigated thoroughly to remove as much loose material as possible and air dried overnight. The teeth were mounted onto aluminium SEM stubs using carbon tabs and silver paint (Agar Scientific) and were left uncoated. Samples were imaged in a scanning electron microscope (Tescan) at $5 \mathrm{kV}$ with a beam current of 300pa. Large area maps were collected of each sample (AZtec, Oxford Instruments) and used for navigation. EDS maps were collected using an Ultim Extreme EDS detector (Oxford Instruments) at multiple locations on each sample using a resolution of 512 pixels, with a pixel dwell of $5 \mu \mathrm{s}$, a process time of 5 and 100 frames in total for each map. The samples were mounted onto AFM compatible pucks and inserted into a Cypher AFM (Oxford Instruments) for imaging in AM-FM mode. SEM images were used to locate the same regions of interest. Imaging was conducted continuously over 60 hours and 1000 scans. The samples were initially placed in acetate buffer $\left(\mathrm{pH} \mathrm{4.3)}\right.$. At the $300^{\text {th }}$ scan the solution was replaced with lactic acid ( $\mathrm{pH}$ 2.2). Following AFM acquisition, the samples were air dried and remounted onto SEM stubs. EM images and EDS maps were collected on the digested samples using the same acquisition parameters as before and on the same areas.

SEM and AFM time course data were correlated and analysed using Relate (Oxford Instruments). Each data set from the time course was aligned using image overlays and key features visible across all the images. Profile lines were used to observe changes in material properties over time from the aligned data sets. EDS maps and EM images were correlated with the before and after AFM data using features such as scratches in the surface for alignment.

Results showed a differential response to acid digestion across all the parameters measured (figure 1). Topographical analysis during acid exposure showed very little effect of the buffer at $\mathrm{pH} \mathrm{4.4,} \mathrm{however,}$ exposure to the lactic acid showed significant differences. Topographical changes were most prevalent between scans 300 and 350 although minor changes were observed all the way up to scan 600. Dissipation measurements showed a large change between scan 300 and 305 and showed no significant changes beyond this. Amplitude measurements showed no significant differences during the time course. SEM measurements correlated with topographical changes observed in the sample.

As correlative microscopy approaches become standard for biological research, it is important that workflows incorporating different types of imaging technology area are established for a range of 
specimen types so that researchers can make informed decisions about sample preparation and imaging hierarchy. The ability to assess measurements in the mechanical properties of the sample were crucial for determining changes within the tooth samples and Relate provided an easy-to-use platform for quantitative analysis of correlated data.
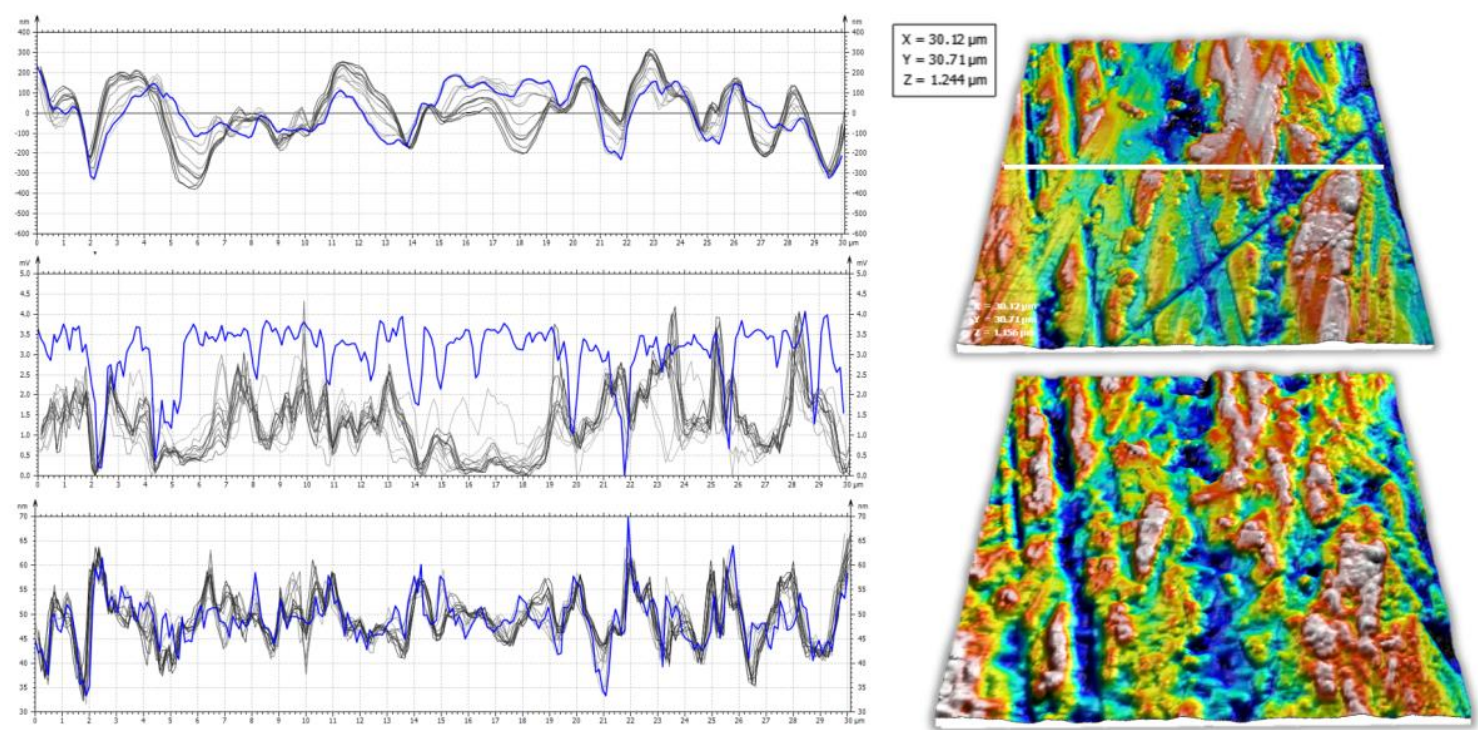

Figure 1. Profile curves for topography (top left), dissipation (middle left) and amplitude (bottom left) and the topographical data at scan 300 and 350 (right). The profile position is indicated by the white line (top right). Profile curves show every other scan from 300 to 350 . The first scan is shown in blue. Changes in the topography are continuous across all the scans compared to a big jump in the dissipation measurements between scan 301 and 305 and no significant changes in the amplitude.

\section{References}

[1] Walter, A., Paul-Gilloteaux, P., Plochberger, B., Sefc, L., Verkade, P., Mannheim, J.G., Slezak, P., Unterhuber, A., Marchetti-Deschmann, M., Ogris, M. and Bühler, K., 2020. Correlated multimodal imaging in life sciences: Expanding the biomedical horizon. Frontiers in Physics, 8, p.47. 